\title{
Frequent loss of SMAD4/DPC4 protein in colorectal cancers
}

\section{R Salovaara, S Roth, A Loukola, V Launonen, P Sistonen, E Avizienyte, P Kristo, H Järvinen, S Souchelnytskyi, M Sarlomo-Rikala, L A Aaltonen}

See end of article for

Gut 2002;51:56-59

authors' affiliations

$\ldots \ldots \ldots \ldots \ldots \ldots$ Background and aims: Loss of DNA sequences from chromosome 18q21 is a major genetic change

Correspondence to:

Dr L A Aaltonen,

Department of Medical

Genetics, Haartman

Institute, PO Box 63 ,

FIN-00014 University of

Helsinki, Finland;

lauri.aaltonen@helsinki.fi.

Accepted for publication

17 September 2001 \begin{abstract}
in colorectal tumorigenesis. Multiple genes have been identified in this area. One of these, DPC4
\end{abstract} (deleted in pancreatic cancer 4, also known as SMAD4), is mutated in a minor subset of colorectal carcinomas as well as in germlines of humans predisposed to colon tumours.

Patients and methods: The involvement of SMAD4 in sporadic colorectal neoplasia was evaluated by immunohistochemistry in 53 unselected cases and 27 cases displaying microsatellite instability.

Results: SMAD4 expression was absent in 20 of $53(38 \%)$ unselected colorectal carcinomas, and reduced in another $15(28 \%)$ cases. However, 26 of 27 cancers displaying microsatellite instability and TGF- $\beta$ IIR mutations were positive for SMAD4 immunostaining.

Conclusions: Loss of SMAD4 expression may play a more prominent role in colon cancer than anticipated based on genetic evidence, but not in mutator phenotype tumours.

M uch work has focused on revealing molecular changes that occur in colorectal neoplasia. These efforts have resulted in a model for human tumour progression depicting a number of key molecular events that drive the malignant process. One of the most important events in colon cancer appears to be loss of genetic material in chromosome 18q. This change is typically found in late adenomas and carcinomas. ${ }^{1}$ A gene termed DCC (deleted in colorectal carcinoma) has been identified through deletion mapping studies, and proposed as the target of the $18 \mathrm{q} 21$ deletions. ${ }^{2}$ More recently, the gene DPC4 (deleted in pancreatic carcinoma 4, also called SMAD4) was identified in $18 \mathrm{q} 21 .^{3}$ While this gene is frequently mutated and deleted in pancreatic carcinomas, less evidence has linked it to colorectal tumorigenesis. ${ }^{4}$ Although a more prominent role of SMAD4 has been implicated in advanced disease, ${ }^{56} S M A D 4$ has not been considered as one of the major players in colonic neoplasia, such as APC, K-RAS, and TP53 genes. ${ }^{7}$ We and others recently found an association between SMAD4 germline defects and human colon tumour susceptibility. ${ }^{8}$ As genes for hereditary cancer are also believed to often play a significant role in the respective sporadic tumours, ${ }^{47}$ we evaluated the role of SMAD4 in colorectal neoplasia using immunohistochemical labelling with a monoclonal SMAD4 antibody. ${ }^{9}$

\section{MATERIALS AND METHODS \\ Cancer specimens}

For SMAD4 immunohistochemical labelling, 53 surgical resection specimens from unselected colorectal carcinoma patients were collected from the files of the Department of Pathology, Haartman Institute, University of Helsinki. In addition, a series of 27 archival colorectal cancer samples displaying microsatellite instability (MSI) or mutator phenotype was available from previous studies. ${ }^{10}{ }^{11}$ The 80 tissue samples were fixed in $10 \%$ neutral buffered formalin and paraffin embedded. Haematoxylin-eosin staining was performed to allow histological characterisation. The tumours were evaluated by two pathologists. All 80 samples contained areas of normal colonic mucosa adjacent to the carcinoma (for Dukes' classification, see tables 1 and 2). The large proportion of Dukes' $\mathrm{C}$ and D cancers in the unselected series is due to patient selection to the Helsinki University Hospital, and coincidence.

Of the 80 colorectal carcinomas analysed for SMAD4 immunohistochemistry, 55 normal/tumour DNA sample pairs were available from previous studies ${ }^{10}{ }^{11} ; 27$ MSI and 28 unselected (mostly microsatellite stable) samples, respectively. These were used for molecular genetic analyses.

\section{Immunohistochemical labelling for SMAD4}

The presence of SMAD4 protein expression was analysed in 80 paraffin embedded specimens by immunohistochemistry. SMAD4 monoclonal antibody raised against a peptide corresponding to amino acids 1-552 representing full length SMAD4 of human origin (Smad4 B-8; sc-7966; Santa Cruz Biotechnology Inc., Santa Cruz, California, USA) was used. The specificity of the antibody has been previously tested, ${ }^{9}$ and the specificity of the present batch was confirmed through western blotting. Western blotting of whole cell lysates of three colon carcinoma cell lines detected SMAD4 expression in two colon carcinoma cell lines; HCT116 and DLD1 displayed the correct sized band without background. No expression was observed in the cell line SW480. The SMAD4 expression status of these cell lines was confirmed using reverse transcription-polymerase chain reaction (RT-PCR) analysis and immunohistochemistry. Human HPRT control amplimer set (Clontech, Palo Alto, California, USA) was used as a positive control for RT-PCR (data not shown).

Unstained $4 \mu \mathrm{m}$ tissue sections from the 80 paraffin embedded cancer specimens were mounted on slides coated with 3-aminopropyl-triethoxy-silane (Sigma, St Louis, Missouri, USA). Sections were deparaffinised in xylene, and rehydrated through a graded alcohol series to distilled water. After deparaffinisation the sections were pretreated in a microwave oven in buffered sodium citrate. For immunohistochemical

Abbreviations: MSI, microsatellite instability; $\mathrm{LOH}$, loss of heterozygosity; RT-PCR, reverse transcription-polymerase chain reaction; TGF- $\beta$ IIR, transforming growth factor $\beta$ type II receptor. 
Table 1 SMAD4 immunohistochemical labelling results for 53 unselected colorectal cancers

\begin{tabular}{lllcccc}
\hline & & \multicolumn{5}{c}{ Dukes' classification } \\
\cline { 5 - 7 } Labelling & Normal mucosa & Carcinoma & A & B & C & D \\
\hline+++ & 29 & 8 & 1 & 3 & 0 & 4 \\
++ & 17 & 10 & 2 & 2 & 2 & 4 \\
+ & 7 & 15 & 0 & 5 & 7 & 3 \\
- & 0 & 20 & 0 & 5 & 11 & 4 \\
Total & 53 & 53 & 3 & 15 & 20 & 15 \\
\hline
\end{tabular}
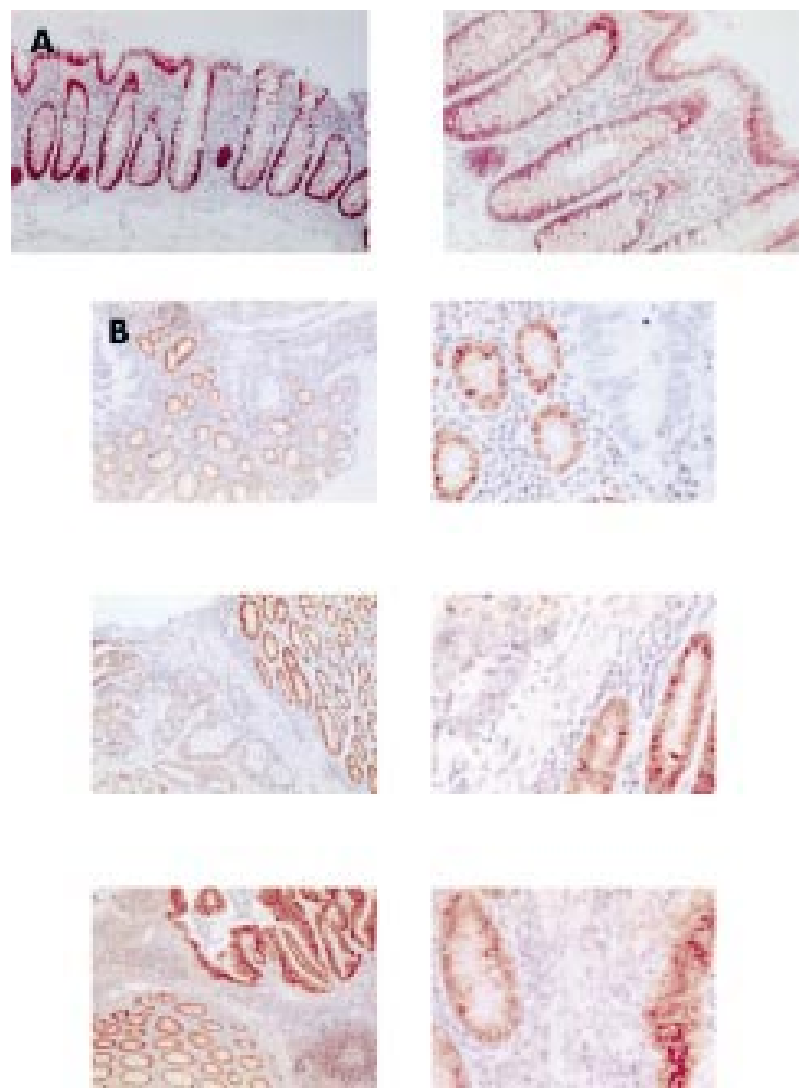

Figure 1 (A) SMAD4 immunohistochemical labelling of the normal colon. Epithelial staining was equally distributed. Nuclear as well as cytoplasmic staining was seen. (B) Examples of SMAD4

immunohistochemical expression in three colon carcinomas with a characteristic positive staining pattern $(+++)$ in normal mucosal epithelial cells. Protein expression was lost (-) (top panels), slightly diminished $(++)$ (middle panels), or maintained (+++) (bottom panels). The lesion with maintained expression displayed microsatellite instability.

analysis, the avidin-biotin complex immunoperoxidase technique was undertaken using the commercial Elite $\mathrm{ABC}$ kit (Vectastain; Vector Laboratories, Burlingame, California, USA).

Endogenous peroxidase was blocked by incubating in hydrogen peroxidase with methanol and then incubating with non-immune horse serum. Also, endogenous biotin was blocked (avidin-biotin blocking kit, SP-2001; Vector Laboratories). Slides were labelled with a 1:2000 dilution of the primary antibody and incubated overnight. Sections were then incubated in biotinylated second antibody and peroxidase labelled avidin-biotin complex for 30 minutes. All dilutions were made using phosphate buffered saline ( $\mathrm{pH} 7.2$ ). Incubations were carried out in humid chambers at room temperature. Staining was visualised using a 3-amino-9- ethylcarbazole (Sigma) solution for 15 minutes at room temperature, and sections were counterstained in Mayer's haematoxylin, rinsed in water, and mounted in aqueous mounting media. SMAD4 antigen expression was analysed by two pathologists. The percentage of positive cells was evaluated and scored as follows: $(-)<5 \%,(+)$ 5-9\%, $(++) 10-34 \%$, and $(+++) \geqslant 35 \%$ (fig 1$)$.

\section{8q21 loss of heterozygosity analysis}

Loss of heterozygosity (LOH) analysis was performed using two fluorescent labelled microsatellite markers (D18S1156 and D18S363). PCR reactions were carried out in a $20 \mu \mathrm{l}$ reaction volume containing $100 \mathrm{ng}$ genomic DNA, $1 \times$ PCR buffer (Applied Biosystems, Foster City, California, USA), $250 \mu \mathrm{M}$ of each dNTP (Finnzymes, Espoo, Finland), $0.5 \mu \mathrm{M}$ of each primer, and 2 units of AmplitaqGold polymerase (Applied Biosystems). $\mathrm{MgCl}_{2}$ concentration was $2 \mathrm{mM}$. The following PCR cycles were used for amplification: 10 minutes at $95^{\circ} \mathrm{C}, 30$ cycles of 45 seconds at $95^{\circ} \mathrm{C}, 45$ seconds at $54^{\circ} \mathrm{C}$, and one minute at $72^{\circ} \mathrm{C}$. Final extension was 10 minutes at $72^{\circ} \mathrm{C}$. PCR products were loaded onto $6 \%$ polyacrylamide gels and run on an ABI PRISM 377 DNA Sequencer (Applied Biosystems). The data were collected automatically and analysed by GeneScan 3.1 software (Applied Biosystems). LOH was scored by calculating the ratio of the peak areas of the constitutional alleles, $\mathrm{L}=\left(\mathrm{a}_{\mathrm{t} 2} \times \mathrm{a}_{\mathrm{n} 1}\right) /\left(\mathrm{a}_{\mathrm{t} 1} \times \mathrm{a}_{\mathrm{n} 2}\right)$. If $\mathrm{L}<0.6$ or $\mathrm{L}>1.67$, one of the tumour alleles had decreased by more than $40 \%$, implicating $\mathrm{LOH}$.

\section{TGF- $\beta$ IIR mutation analysis}

The polyA tract in the coding region of the transforming growth factor $\beta$ type II receptor (TGF- $\beta$ IIR) gene was scrutinised for deletions by PCR amplification using fluorescent labelled primers and subsequent fragment analysis by an automated sequencer. The PCR reactions were carried out in a $10 \mu \mathrm{l}$ reaction volume including $100 \mathrm{ng}$ genomic DNA, $1 \times \mathrm{PCR}$ reaction buffer (PE/ABI), $200 \mu \mathrm{M}$ of each dNTP (Finnzymes), $0.3 \mu \mathrm{M}$ of each primer, and 1.5 units of AmplitaqGOLD polymerase (PE/ $\mathrm{ABI})$. $\mathrm{MgCl}_{2}$ concentration was $1.5 \mathrm{mM}$. The following PCR cycles were used for amplification: 10 minutes at $94^{\circ} \mathrm{C}, 28$ cycles of 30 seconds at $94^{\circ} \mathrm{C}, 75$ seconds at $55^{\circ} \mathrm{C}$, and 30 seconds at $72^{\circ} \mathrm{C}$. Final extension was 10 minutes at $72^{\circ} \mathrm{C}$. The forward (F) and reverse (R) primers were: F: $5^{\prime}$-CTT TATTCTGGAAGATGCTG; R: 5'-GAAGAAAGTCTCACCAGGC.

\section{RESULTS AND DISCUSSION SMAD4 immunostaining in unselected colorectal carcinomas}

Normal colonic mucosa displayed positive staining in all cases. Seven of 53 were scored as $(+)$ and the remaining 46 as $(++)$ or $(+++)$ (table 1$)$. Nuclear as well as cytoplasmic staining was seen, similar to the pattern recently reported. ${ }^{9}$ Epithelial staining was typically equally distributed (fig 1A) but occasionally stronger staining was observed in the bottom of the crypts. This differs from a recent study using a different SMAD4 antibody where stronger staining was detected in the apical side. $^{12}$ 
Table 2 SMAD4 immunohistochemical labelling results for 27 colorectal cancers with microsatellite instability

\begin{tabular}{llccccc}
\hline & & \multicolumn{3}{l}{ Dukes' classification } \\
\cline { 4 - 7 } Labelling & Normal mucosa & Carcinoma & A & B & C & D \\
\hline+++ & 18 & 16 & 3 & 7 & 5 & 1 \\
++ & 8 & 10 & 0 & 7 & 1 & 2 \\
+ & 1 & 0 & 0 & 0 & 0 & 0 \\
- & 0 & 1 & 0 & 1 & 0 & 0 \\
Total & 27 & 27 & 3 & 15 & 6 & 3 \\
\hline
\end{tabular}

Compared with results from normal mucosa, SMAD4 staining was frequently absent in carcinomas $(p<0.0001$, Fisher's exact test). Sixty six per cent (35 of 53) of unselected colorectal adenocarcinomas displayed absent (-) (20 of 53, $38 \%)$ or decreased $(+)(15$ of $53,28 \%)$ staining. In 18 of 53 $(34 \%)$ carcinomas, SMAD4 expression comparable with normal tissue expression $(++$ to +++ ) was observed (table 1, fig $1 B$ )

\section{Correlation between SMAD4 immunostaining and microsatellite marker analyses}

Twenty seven samples showing MSI were originally selected for SMAD4 immunostaining based on previous MSI data. ${ }^{10}{ }^{11}$ MSI tumours frequently display inactivating mutations of the $T G F$ - $\beta I I R$ gene; deletions in a polyA microsatellite tract in the coding region of TGF- $\beta I I R$ in particular are characteristic of these lesions. ${ }^{13}$ In agreement with previous observations, in our series 21 of 25 (84\%) tumours available for analysis displayed a protein truncating mutation in the TGF- $\beta I I R$ polyA tract (not shown). Unlike most colorectal cancers, MSI tumours are typically diploid, and rarely display gross chromosomal rearrangements, such as deletions. ${ }^{14}{ }^{15}$ We next examined SMAD4 immunostaining in the 27 MSI colorectal cancers. The immunostaining results in this series of tumours were strikingly different from the unselected carcinomas $(p<0.0009$, Fisher's exact test): 26 of 27 (96\%) MSI cancers had SMAD4 expression, comparable with normal tissue, while one $(4 \%)$ displayed no staining (table 2 ).

LOH data were created with 18q21 microsatellite markers D18S1156 and D18S363 (see methods). This analysis focused on 28 unselected tumours (table 3 ). Also, MSI samples were included in the analysis but $88 \%$ of the results were uninformative because of aberrant MSI alleles or homozygosity (data not shown). LOH status of the unselected tumours did not have an effect on the SMAD4 immunostaining results (table 3). Most unselected tumours ( 15 of 22 (68\%) informative cases) displayed evidence of deletions. Deletions were equally present in tumours with reduced $(-$ or +$)$ and normal $(++$ or +++$)$ SMAD4 staining (table 3$)$.

Previous studies have confirmed the role of SMAD4 in colorectal tumorigenesis in a subset of these lesions. ${ }^{4}$ The extent of this contribution however has been more ambiguous. While the first estimates of SMAD4 mutation frequency in colon cancer were low, ${ }^{4}$ it seems that in metastasised disease the mutation rate is higher. ${ }^{5}$ The frequency of SMAD4 mutations has been found to increase from $0 \%$ in adenomas to $10 \%$ in carcinomas, and up to $35 \%$ in invasive carcinomas with metastases. ${ }^{5}$ A similar but not significant tendency towards loss of SMAD4 protein in metastatic disease can also be observed in the unselected tumour series; $43 \%$ in Dukes' $\mathrm{C}$ and D cancers combined. That only four of 15 (27\%) Dukes' D carcinomas were SMAD4 negative may be due to the small numbers. SMAD4 was relatively frequently lost in localised disease also (28\% in Dukes' A and B) (table 1). These values are higher than those reported by Maitra and colleagues ${ }^{16}$ using the same antibody. The difference may arise from a different scoring
Table $318 q 21 \mathrm{LOH}$ analysis of the 28 unselected colorectal carcinomas where normal/tumour DNA pairs were available.

\begin{tabular}{|c|c|c|c|}
\hline Case & D18S1156 & D18S363 & $\mathrm{IHC}$ \\
\hline C964 & - & $\mathrm{LOH}$ & $\mathrm{Neg}$ \\
\hline C972 & $\mathrm{LOH}$ & $\mathrm{LOH}$ & Neg \\
\hline C986 & - & $N$ & Neg \\
\hline C978 & - & $\mathrm{N}$ & $\mathrm{Neg}$ \\
\hline C989 & - & $\mathrm{LOH}$ & $\mathrm{Neg}$ \\
\hline C1038 & $\mathrm{N}$ & - & Neg \\
\hline C1051 & - & - & $\mathrm{Neg}$ \\
\hline C1079 & - & $\mathrm{N}$ & Neg \\
\hline C1086 & $\mathrm{N}$ & $N$ & $\mathrm{Neg}$ \\
\hline C977 & $\mathrm{N}$ & $\mathrm{LOH}$ & + \\
\hline C981 & $\mathrm{LOH}$ & $\mathrm{LOH}$ & + \\
\hline C982 & - & $\mathrm{LOH}$ & + \\
\hline C984 & - & - & + \\
\hline C1064 & - & - & + \\
\hline C1084 & $\mathrm{LOH}$ & - & + \\
\hline $\mathrm{C} 1091$ & $\mathrm{~N}$ & $\mathrm{LOH}$ & + \\
\hline C1089 & $\mathrm{LOH}$ & $\mathrm{N}$ & + \\
\hline $\mathrm{C} 1088$ & - & $\mathrm{LOH}$ & + \\
\hline C980 & - & $N$ & ++ \\
\hline C 1047 & $\mathrm{LOH}$ & - & ++ \\
\hline C1048 & - & - & ++ \\
\hline C1066 & - & - & ++ \\
\hline C1083 & - & - & ++ \\
\hline C965 & - & $\mathrm{LOH}$ & +++ \\
\hline C1074 & $N$ & $\mathrm{LOH}$ & +++ \\
\hline C1077 & $\mathrm{LOH}$ & MSI & +++ \\
\hline $\mathrm{C} 1080$ & $N$ & $N$ & +++ \\
\hline C1085 & - & $\mathrm{LOH}$ & +++ \\
\hline
\end{tabular}

-, uninformative or failed amplifications; $\mathrm{N}$, heterozygous unchanged loci; $\mathrm{LOH}$, loss of heterozygosity; MSI, microsatellite instability; IHC, immunohistochemistry.

system, in addition to technical matters and true differences between the respective study materials. In our study, tumours displaying positive staining in less than $5 \%$ of tumour cells were classified as negative whereas in the study of Maitra and colleagues ${ }^{16}$ any positivity was taken into account.

Our study suggests that SMAD4 expression is more frequently lost during colorectal tumorigenesis than anticipated previously (almost $40 \%$ of our unselected series). In addition, reduced expression was detected in $28 \%$. The latter may be of significance as it is possible that heterozygous loss of SMAD4 and the consequent decrease in expression contributes to malignant growth. ${ }^{17}$

Prominent expression of SMAD4 in MSI colorectal cancers is conceivable as both SMAD4 and TGF- $\beta$ IIR contribute to tumour suppressive effects as members of the TGF- $\beta$ signalling pathway ${ }^{18}$ although BMP signalling may also contribute to colorectal tumorigenesis through SMAD4. ${ }^{19}$ While genomic deletions and somatic mutations explain for the most part loss of SMAD4 in colon cancers, the mechanism of complete SMAD4 loss in 38\% of an unselected tumour series and lack of correlation between $\mathrm{LOH}$ and reduction of 
staining remain somewhat obscure. Possible additional inactivation mechanisms include promoter area mutations and epigenetic changes such as hypermethylation. In a separate work, we found no evidence of these in SMAD4. ${ }^{20}$ Downregulated expression through transcription factors secondary to genetic events elsewhere, similar to recent findings on the background of c-MYC overexpression in colorectal cancer due to $A P C$ mutations, ${ }^{21}$ is also possible.

While we cannot exclude the possibility that decreased SMAD4 levels are simply due to frequent deletions in 18q21 targeted to other tumour suppressor genes, we propose that SMAD4 loss is likely to be a key event in colorectal tumorigenesis. Firstly, SMAD4 is somatically inactivated in a number of colorectal tumours. In addition to deletions, frameshift mutations as well as point mutations that have functional consequences have been described, convincingly demonstrating that specific genetic changes occur in SMAD4 in tumorigenesis. ${ }^{317} 182223$ Secondly, the role of SMAD4 in intestinal tumorigenesis has been demonstrated through studies linking germline SMAD4 defects to intestinal tumour susceptibility, in animal models as well as in humans. ${ }^{823}$ Thirdly, SMAD4 function has been elucidated in great detail, although functions as yet unknown may exist. The present data provide a firm base for the hypothesis that SMAD4 is a key molecule in pathways involved in cell growth control. ${ }^{18}$ Fourthly, our data show that a decrease and complete loss of SMAD4 expression is a frequent phenomenon in colorectal carcinogenesis. The data presented here suggest a more prominent role of SMAD4 in colorectal cancer than anticipated based on genetic evidence.

\section{ACKNOWLEDGEMENTS}

We thank Siv Lindroos, Sinikka Lindh, Kirsi Laukkanen, Inga-Lill Svedberg, Annika Lahti, and Anitta Hottinen for technical assistance, and Heikki Joensuu for advice on statistics. Supported by grants from the Academy of Finland (44870, Finnish Centre of Excellence Programme 2000-2005), Finnish Cancer Societies, Sigrid Juselius Foundation, Emil Aaltonen Foundation, Helsinki University Central Hospital, Biocentrum Helsinki, and the European Commission Contract BMH4-CT98-3865.

\section{Authors' affiliations}

R Salovaara, Department of Medical Genetics and Department of Pathology, Haartman Institute, PO Box 63, FIN-00014 University of Helsinki, Finland

S Roth, A Loukola, V Launonen, E Avizienyte, P Kristo,, Department of Medical Genetics, Haartman Institute, PO Box 63, FIN-00014 University of Helsinki, Finland

P Sistonen, Finnish Red Cross Blood Transfusion Service, Kivihaantie 7, FIN-00310 Helsinki, Finland

H Järvinen, Second Department of Surgery, Helsinki University Central Hospital, PO Box 340, FIN-00029 Helsinki, Finland

S Souchelnytskyi, Ludwig Institute for Cancer Research, PO Box 595,

S-751 24 Uppsala, Sweden

M Sarlomo-Rikala, Department of Pathology, Haartman Institute, PO
Box 63, FIN-00014 University of Helsinki, Finland

L A Aaltonen, Department of Medical Genetics, Haartman Institute, PO Box 63, FIN-00014 University of Helsinki, Finland, and Department of

Oncology, Helsinki University Central Hospital, PO Box 180, FIN-00029 Helsinki, Finland

\section{REFERENCES}

1 Vogelstein B, Fearon ER, Hamilton SR, et al. Genetic alterations during colorectal tumor development. N Engl J Med 1988;319:525-32.

2 Fearon E, Cho K, Nigro J, et al. Identification of a chromosome 18q gene that is altered in colorectal cancers. Science 1990;247:49-56.

3 Hahn SA, Schutte M, Hoque ATMS, et al. DPC4, a candidate tumor suppressor gene at human chromosome 18q21.1. Science 1996:271:350-3.

4 Thiagalingam S, Lengaver $\mathrm{C}$, Leach FS, et al. Evaluation of candidate tumour suppressor genes on chromosome 18 in colorectal cancers. Nat Genet 1996;13:343-6.

5 Miyaki $M$, liiima T, Konishi $M$, et al. Higher frequency of Smad4 gene mutation in human colorectal cancer with distant metastasis. Oncogene 1999;20:3098-103.

6 Koyama $M$, Ito $M$, Nagai $H$, et al. Inactivation of both alleles of the DPC4/SMAD4 gene in advanced colorectal cancers: identification of seven novel somatic mutations intumours from Japanese patients. Mutat Res 1999;406:71-7.

7 Kinzler KW, Vogelstein B. Lessons from hereditary colorectal cancer Cell 1996;280: 159-70

8 Howe JR, Roth S, Ringold JC, et al. Mutations in the SMAD4/DPC4 gene in juvenile polyposis. Science 1998;280:1086-8.

9 Wilentz RE, Su GH, Dai JL, et al. Immunohistochemical labeling for Dpc4 mirrors genetic status in pancreatic adenocarcinomas. A new marker of DPC4 inactivation. Am J Pathol 2000;156:37-43

10 Aaltonen LA, Salovaara R, Kristo $P$, et al. Incidence of hereditary nonpolyposis colorectal cancer, and molecular screening for the disease. N Engl J Med 1998;338:1481-7.

11 Salovaara R, Loukola A, Kristo P, et al. Population-based molecular detection of hereditary nonpolyposis colorectal cancer. J Clin Oncol 2000;18:2193-200.

12 Korchynskyi O, Landström M, Stoika R, et al. Expression of SMAD proteins in human colorectal cancers. Int J Cancer 1999;82:197-202.

13 Markowitz S, Wang J, Myeroff L, et al. Inactivation of the type II TGF-beta receptor in colon cancer cells with microsatellite instability. Science 1995;268:1336-8.

14 Aaltonen LA, Peltomäki $\mathrm{P}$, Leach FS, et al. Clues to the pathogenesis of familial colorectal cancer. Science 1993;260:812-16.

15 Thibodeau SN, Bren G, Schaid D. Microsatellite instability in cancer of the proximal colon. Science 1993;260:816-19.

16 Maitra A, Molberg K, Albores-Saavedra J, et al. Loss of Dpc4 expression in colonic adenocarcinomas correlates with the presence of metastatic disease. Am J Pathol 2000;157: 1 105-1 1 .

17 Xiaoling Xu, Brodie SG, Yang X, et al. Haploid loss of the tumor suppressor Smad4/Dpc4 initiates gastric polyposis and cancer in mice. Oncogene 2000;19:1868-74

18 Heldin CH, Miyazono K, ten Dijke P. TGF-beta signaling from cell membrane to nucleus through SMAD proteins. Nature 1997;390:465-71

19 Howe JR, Bair JL, Sayed MG, et al. Germline mutations of the gene encoding bone morphogenetic protein receptor $1 \mathrm{~A}$ in juvenile polyposis. Nat Genet 2001;28:184-7.

20 Roth S, Laiho P, Salovaara R, et al. No SMAD4 hypermethylation in colorectal cancer. Br J Cancer 2000;83:1015-19.

21 He TC, Sparks AB, Rago C, et al. Identification of c-MYC as a target of the APC pathway. Science 1998;281:1509-12

22 Shi Y, Hata A, Lo RS, et al. A structural basis for mutational inactivation of the fumour suppressor Smad4. Nature 1997;388:87-93.

23 Takaku K, Oshima M, Miyoshi $\mathrm{H}$, et al. Intestinal tumorigenesis in compound mutant mice of both Dpc4 (Smad4) and Apc genes. Cell 1998;92:645-56. 